\title{
2+1 flavor lattice QCD simulation with $O(a)$-improved Wilson quarks
}

PACS-CS Collaboration : N. Ukita ${ }^{* a \dagger}$, S. Aoki ${ }^{b, c}$, N. Ishii ${ }^{a}$, K.-I. Ishikawa ${ }^{d}$, N. Ishizuka ${ }^{a, b}$, T. Izubuchi ${ }^{c, e}$, D. Kadoh ${ }^{a}$, K. Kanaya ${ }^{b}$, Y. Kuramashi ${ }^{a, b}$, Y. Namekawa ${ }^{a}$, M. Okawa ${ }^{d}$, Y. Taniguchi ${ }^{a, b}$, A. Ukawa ${ }^{a, b}$, T. Yoshié ' $^{a, b}$

${ }^{a}$ Center for Computational Sciences, University of Tsukuba, Tsukuba, Ibaraki 305-8577, Japan

${ }^{b}$ Graduate School of Pure and Applied Sciences, University of Tsukuba, Tsukuba, Ibaraki 305-8571, Japan

${ }^{c}$ Riken BNL Research Center, Brook-haven National Laboratory, Upton, New York 11973, USA

${ }^{d}$ Graduate School of Sciences, Hiroshima University, Higashi-Hiroshima, Hiroshima 739-8526, Japan

${ }^{e}$ Institute for Theoretical Physics, Kanazawa University, Kanazawa, Ishikawa 920-1192, Japan

We present simulation details and results for the light hadron spectrum in $N_{f}=2+1$ lattice QCD with the nonperturbatively $O(a)$-improved Wilson quark action and the Iwasaki gauge action. Simulations are carried out at a lattice spacing of $0.09 \mathrm{fm}$ on a $(2.9 \mathrm{fm})^{3}$ box using the PACS-CS computer. We employ the Lüscher's domain-decomposed HMC algorithm with several improvements to reduce the degenerate up-down quark mass toward the physical value. So far the resulting pseudoscalar meson mass is ranging from $702 \mathrm{MeV}$ down to $156 \mathrm{MeV}$. We discuss on the stability and the efficiency of the algorithm. The light harden spectrum extrapolated at the physical point is compared with the experimental values. We also present the values of the quark masses and the pseudoscalar meson decay constants.

The XXVI International Symposium on Lattice Field Theory

July 14-192008

Williamsburg, Virginia, USA

\footnotetext{
* Speaker.

${ }^{\dagger}$ E-mail: ukita@ccs.tsukuba.ac.jp
} 


\section{Introduction}

The PACS-CS (Parallel Array Computer System for Computational Sciences) project 11,2 3, 4, 5, 6, 7, 8] aims at $N_{f}=2+1$ lattice QCD calculations at the physical point to remove the most troublesome systematic errors associated with the chiral extrapolations. So far our simulation points cover from $67 \mathrm{MeV}$ to $3.5 \mathrm{MeV}$ for the degenerate up-down quark mass with the strange quark mass fixed around the physical value. The reduction of $m_{\mathrm{ud}}$ down to $10 \mathrm{MeV}$ is achieved by the domain-decomposed Hybrid Monte Carlo (DDHMC) algorithm with the replay trick [9 10]. For the simulation at $m_{\mathrm{ud}}=3.5 \mathrm{MeV}$ we incorporate some algorithmic improvements such as the mass preconditioning [11, 12], the chronological inverter[13] and the deflation teqnique[14] which make simulations stable and contribute to reduce the simulation cost. For the strange quark part we employ the UV-filtered Polynomial Hybrid Monte Carlo (UVPHMC) algorithm[15].

In this report we present the simulation details and some eminent results for the hadron spectrum. Chiral analyses on the pseudoscalar meson sector with the SU(2) and SU(3) chiral perturbation theories and calculation of the charm quark systems with the relativistic heavy quark action are given in separate reports 16, 17.

\section{Simulation details}

We employ the $O(a)$-improved Wison quark action with a nonperturbative improvement coefficient $c_{\mathrm{sw}}=1.715[18]$ and the Iwasaki gauge action[19]. All the simulations are carried out on a $32^{3} \times 64$ lattice at $\beta=1.90$ corresponding to the lattice spacing of $a=0.09 \mathrm{fm}$. Table 1 summarizes our simulation parameters. We choose combinations of the hopping parameters $\left(\kappa_{\mathrm{ud}}, \kappa_{\mathrm{s}}\right)$ based on the previous CP-PACS/JLQCD results 20, 21] except $\left(\kappa_{\mathrm{ud}}, \kappa_{\mathrm{s}}\right)=(0.137785,0.13660)$ which is adjusted at the physical point with the use of the PACS-CS results in an early stage 8 , 4 . The physics results at $\left(\kappa_{\mathrm{ud}}, \kappa_{\mathrm{s}}\right)=(0.137785,0.13660)$ is presented in Ref. [7].

The DDHMC algorithm is implemented for the up-down quark by domain-decomposing the full lattice with an $8^{4}$ block size as a preconditioner for HMC. The domain-decomposition factorizes the up-down quark determinant into the UV and the IR parts geometrically. As a result we have the gauge force and the up-down quark force with the UV and the IR parts in the molecular dynamics evolution. The reduction of the simulation cost is achieved by applying the multiple time scale integrator [22] to these three forces. We find that the relative magnitude of the force terms is given as follows:

$$
\left\|F_{\mathrm{G}}\right\|:\left\|F_{\mathrm{UV}}\right\|:\left\|F_{\mathrm{IR}}\right\| \approx 16: 4: 1
$$

where $F_{\mathrm{G}}$ denotes the gauge part and $F_{\mathrm{UV}, \mathrm{IR}}$ for the UV and the IR parts of the up-down quark. The associated step sizes $\delta \tau_{\mathrm{G}}, \delta \tau_{\mathrm{UV}}, \delta \tau_{\mathrm{IR}}$ are chosen such that

$$
\delta \tau_{\mathrm{G}}|| F_{\mathrm{G}}\left\|\approx \delta \tau_{\mathrm{UV}}\right\| F_{\mathrm{UV}}\left\|\approx \delta \tau_{\mathrm{IR}}\right\| F_{\mathrm{IR}} \|
$$

These step sizes are controlled by three integers $N_{0}, N_{1}, N_{2}$ as $\delta \tau_{\mathrm{G}}=\tau / N_{0} N_{1} N_{2}, \delta \tau_{\mathrm{UV}}=\tau / N_{1} N_{2}$, $\delta \tau_{\text {IR }}=\tau / N_{2}$ with $\tau$ the trajectory length. We fix $N_{0}=N_{1}=4$ in our all simulations. The value of $N_{2}$ is adjusted to make the simulation stable. The threshold for the replay trick is chosen to be $d H=2$. 
Table 1: Summary of simulation parameters. Quark masses are perturbatively renormalized in the $\overline{\mathrm{MS}}$ scheme at the scale of $\mu=1 / a$. The replay trick is applied for the case of $d H>2$. MD time is the number of trajectories multiplied by the trajectory length $\tau$. CPU time for unit $\tau$ is measured on 256 nodes of the PACS-CS computer.

\begin{tabular}{lllllll|l}
\hline$\kappa_{\mathrm{ud}}$ & 0.13700 & 0.13727 & 0.13754 & 0.13754 & 0.13770 & 0.13781 & 0.137785 \\
$\kappa_{\mathrm{s}}$ & 0.13640 & 0.13640 & 0.13640 & 0.13660 & 0.13640 & 0.13640 & 0.13660 \\
\hline HMC & DD & DD & DD & DD & DD & MP & MP2 \\
$\tau$ & 0.5 & 0.5 & 0.5 & 0.5 & 0.25 & 0.25 & 0.25 \\
$\left(N_{0}, N_{1}, N_{2}, N_{3}, N_{4}\right)$ & $(4,4,10)$ & $(4,4,14)$ & $(4,4,20)$ & $(4,4,28)$ & $(4,4,16)$ & $(4,4,4,6)$ & $(4,4,2,4,4)$ \\
& & & & & & $(4,4,6,6)$ & \\
$\rho_{1}$ & - & - & - & - & - & 0.9995 & 0.9995 \\
$\rho_{2}$ & - & - & - & - & - & - & 0.9990 \\
$N_{\text {poly }}$ & 180 & 180 & 180 & 220 & 180 & 200 & 220 \\
replay trick & on & on & on & on & on & off & off \\
rate of $d H>2$ & $0 \%$ & $0.08 \%$ & $0.5 \%$ & $0.1 \%$ & $3 \%$ & $2.8 \%$ & $0.9 \%$ \\
MD time & 2000 & 2000 & 2250 & 2000 & 2000 & 990 & 950 \\
CPU time $[\mathrm{hrs}]$ & 0.29 & 0.44 & 1.3 & 1.1 & 2.7 & 7.1 & 6.0 \\
$m_{\mathrm{ud}}^{\overline{M S}}[\mathrm{MeV}]$ & $66.8(7)$ & $45.3(5)$ & $24.0(3)$ & $21.0(3)$ & $12.3(2)$ & $3.5(2)$ & $3.5(1)$ \\
$m_{\pi}[\mathrm{MeV}]$ & $702(7)$ & $570(6)$ & $411(4)$ & $385(4)$ & $296(3)$ & $156(2)$ & $164(4)$ \\
\hline
\end{tabular}

For the strange quark we employ the UVPHMC algorithm, where the domain-deconposition is not used. The polynomial order $N_{\text {poly }}$ for the UVPHMC algorithm is adjusted to keep high acceptance rate for the global Metropolis test at the end of each trajectory. Based on our observation of $\left\|F_{\mathrm{S}}\right\| \approx\left\|F_{\mathrm{IR}}\right\|$ for the strange quark force, we set $\delta \tau_{\mathrm{s}}=\delta \tau_{\mathrm{IR}}$. Calclation of the IR force requires the inversion of the Wilson-Dirac operator on the full lattice, which is carried out by the SAP (Schwarz alternative procedure) preconditioned GCR algorithm. We use the SSOR preconditioned GCR algorithm for the UV part. These preconditionings are accelerated with the single precision arithmetic. We employ the stopping condition $|D x-b| /|b|<10^{-9}$ for the force calculation and $10^{-14}$ for the Hamiltonian, which guarantees the reversibility of the molecular dynamics trajectories to high precision. The DDHMC algorithm for the up-down quark works efficiently for $\kappa_{\mathrm{ud}} \leq 0.13770$.

As we reduce the up-down quark mass, the increasing fluctuations of the $\left\|F_{\mathrm{IR}}\right\|$ make the simulation unstable. To suppress the fluctuations of $\left\|F_{\mathrm{IR}}\right\|$, we incorporate the mass preconditioning for the IR part (MPDDHMC), which splits the IR force $F_{\mathrm{IR}}$ into $F_{\mathrm{IR}}^{\prime}$ and $\tilde{F}_{\mathrm{IR}}$ by introducing a new hopping parameter $\kappa_{\text {ud }}^{\prime}=\rho_{1} \kappa_{\text {ud }}$ with $\rho_{1}$ less than unity. In the MPDDHMC algorithm we need four integers $\left(N_{0}, N_{1}, N_{2}, N_{3}\right)$ to controll the four step sizes $\delta \tau_{\mathrm{G}}, \delta \tau_{\mathrm{UV}}, \delta \tau_{\mathrm{IR}}^{\prime}, \delta \tilde{\tau}_{\mathrm{IR}} . N_{2}, N_{3}$ and $\rho_{1}$ are adjusted to reduce the fluctuations of $\left\|F_{\mathrm{IR}}^{\prime}\right\|$ and $\left\|\tilde{F}_{\mathrm{IR}}\right\|$. We choose $\delta \tau_{\mathrm{s}}=\delta \tau_{\mathrm{IR}}^{\prime}$ for the strange quark force in the UVPHMC algorithm.

For the run at $\kappa_{\mathrm{ud}}=0.137785$ further mass preconditioning is applied to the shifted IR force $F_{\mathrm{IR}}^{\prime}$, which is divided into $F_{\mathrm{IR}}^{\prime \prime}$ and $\tilde{F}_{\mathrm{IR}}^{\prime}$ using an additional hopping parameter $\kappa_{\mathrm{ud}}^{\prime \prime}=\rho_{2} \kappa_{\mathrm{ud}}^{\prime}=\rho_{2} \rho_{1} \kappa_{\mathrm{ud}}$ with $\rho_{2}$ less than unity. We refer to this algorithm as MP2DDHMC because of two-level of mass 
preconditioning. In this case five step sizes $\delta \tau_{\mathrm{G}}, \delta \tau_{\mathrm{UV}}, \delta \tau_{\mathrm{IR}}^{\prime \prime}, \delta \tilde{\tau}_{\mathrm{IR}}^{\prime}, \delta \tilde{\tau}_{\mathrm{IR}}$ are controlled by five integers $\left(N_{0}, N_{1}, N_{2}, N_{3}, N_{4}\right)$. We adjust the values of $N_{2}, N_{3}, N_{4}$ and $\rho_{1}, \rho_{2}$ to keep stable the fluctuations of $\left\|F_{\mathrm{IR}}^{\prime \prime}\right\|,\left\|\tilde{F}_{\mathrm{IR}}^{\prime}\right\|,\left\|\tilde{F}_{\mathrm{IR}}\right\| . \delta \tau_{\mathrm{s}}$ is equal to $\delta \tau_{\mathrm{IR}}^{\prime \prime}$.

For the MPDDHMC and the MP2DDHMC algorithms the inversion of the Wilson-Dirac operator on the full lattice is composed of three steps. Firstly, we prepare the initial solutions employing the chronological guess with the last 16 solutions. Secondly, we apply a nested BiCGStab solver consisting of the outer solver and the inner one. The latter with single precision arithmetic works as a preconditioner for the former operated with double precision. We employ a stringent stopping condition $|D x-b| /|b|<10^{-14}$ for the outer solver and an automatic tolerance control ranging from $10^{-3}$ to $10^{-6}$ for the inner solver. Thirdly, the nested BiCGStab solver is replaced by the GCRO-DR (Generalized Conjugate Residual with implicit inner Orthogonalization and Deflated Restarting) algorithm, once the inner BiCGStab solver becomes stagnant during the inversion of the Wilson-Dirac operator.

In Figs. 1 and 2 we show the $d H$ and the force histories at $\kappa_{\mathrm{ud}}=0.13727$ and 0.13770 with the DDHMC algorithm and those at $\kappa_{\mathrm{ud}}=0.13781$ with the MPDDHMC algorithm. The time histories at $\kappa_{\mathrm{ud}}=0.13727$ are quite stable, whereas the $\kappa_{\mathrm{ud}}=0.13770$ case shows the spike-like fluctuations of the IR force at a few $\%$ rate of trajectries. For the $\kappa_{\mathrm{ud}}=0.13781$ run we observe that the MPDDHMC algorithm succeeds in reducing the fluctuations of the IR forces $F_{\mathrm{IR}}^{\prime}$ and $\tilde{F}_{\mathrm{IR}}$.


Figure 1: $d H$ histories for $\left(\kappa_{\mathrm{ud}}, \kappa_{\mathrm{s}}\right)=(0.13727,0.13640),(0.13770,0.13640)$ and $(0.13781,0.13640)$ from the left.
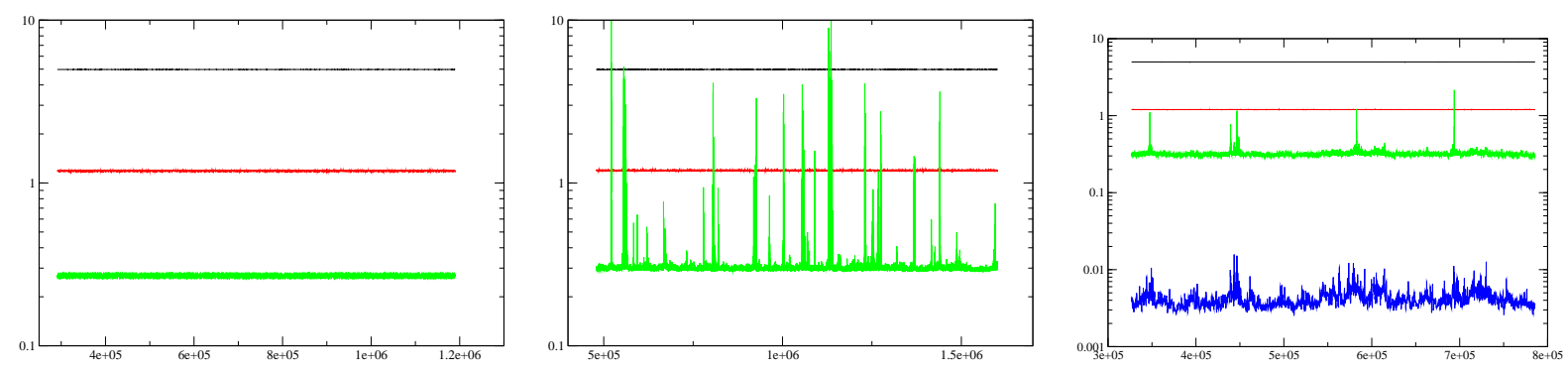

Figure 2: Force histories for $\left(\kappa_{\mathrm{ud}}, \kappa_{\mathrm{s}}\right)=(0.13727,0.13640),(0.13770,0.13640)$ and $(0.13781,0.13640)$ from the left. In the left and middle figures black, red and green lines denote $F_{\mathrm{G}}, F_{\mathrm{UV}}$ and $F_{\mathrm{IR}}$, respectively, with the DDHMC algorithm, In the right figure black, red, green and blue lines are for $F_{\mathrm{G}}, F_{\mathrm{UV}}, F_{\mathrm{IR}}^{\prime}$ and $\tilde{F}_{\mathrm{IR}}$, respectively, with the MPDDHMC algorithm. 


\section{Hadron spectrum}

We measure hadron correlators at every 10 trajectories for $\kappa_{\mathrm{ud}} \leq 13770$ and 20 trajectories for $\kappa_{\mathrm{ud}} \geq 13781$. Light hadron masses are extracted from single exponential $\chi^{2}$ fits to the correlators with an exponentially smeared source and a local sink. In order to increase the statistics we take four source points with different time slices for $\kappa_{\mathrm{ud}} \geq 0.13754$. They are averaged on each configuration before the jackknife analysis. This reduces the statistical errors by typically $20-40 \%$ for the vector meson and the baryon masses and less than $20 \%$ for the pseudoscalar meson masses compared to a single source point. Statistical errors are estimated by the jackknife method. Figure 3 shows the binsize dependence of the error for the pion mass and the " $\eta_{s s}$ " meson mass. We observe that the magnitude of the error reaches a plateau after 100-200 MD time. This feature seems almost independent of the quark mass. Since similar binsize dependences are found for other particle types, we choose a binsize of $250 \mathrm{MD}$ time at $\kappa_{\mathrm{ud}}<0.13770$. At $\kappa_{\mathrm{ud}}=0.13781$ we employ a binsize of $110 \mathrm{MD}$ time due to the lack of statistics.
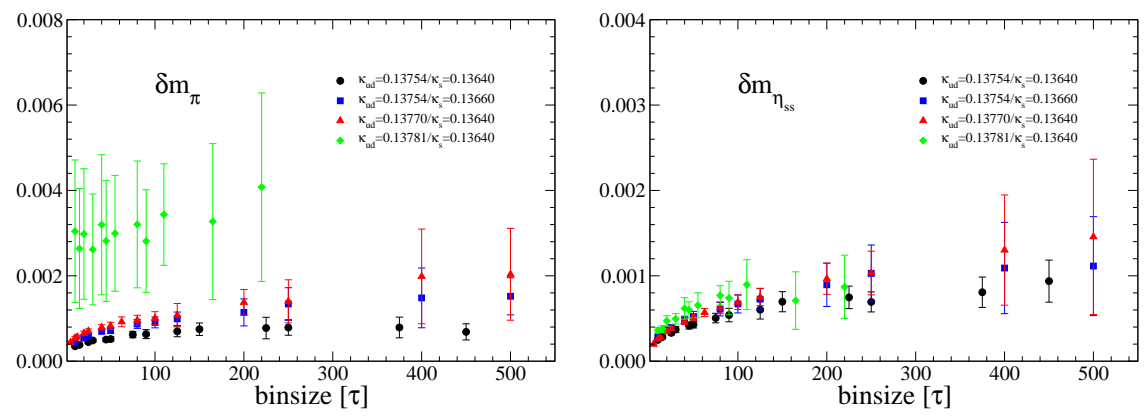

Figure 3: Binsize dependence of the magnitude of error for $m_{\pi}$ (left) and $m_{\eta_{\mathrm{ss}}}$ (right) at $\kappa_{\mathrm{ud}} \geq 0.13754$
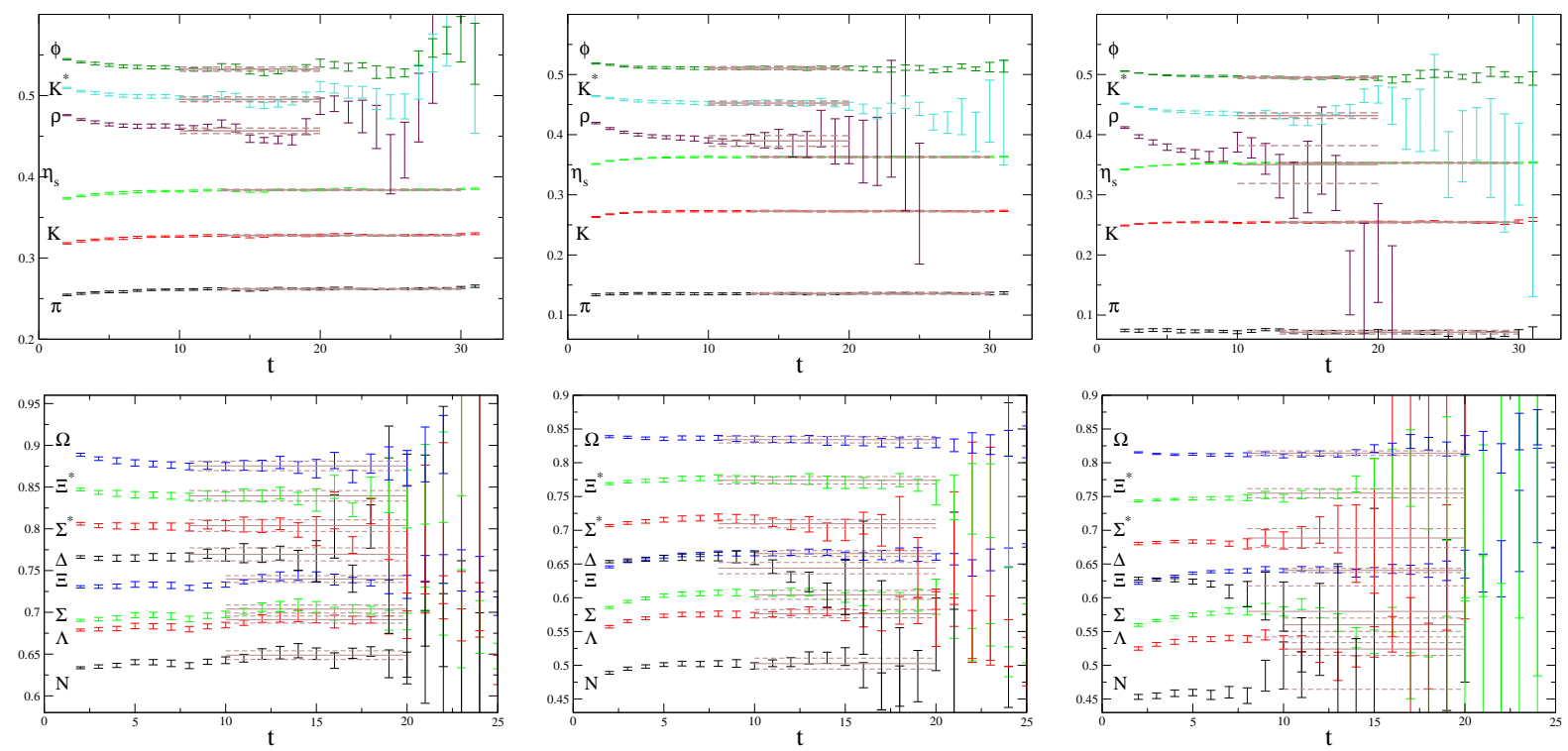

Figure 4: Effective masses for the mesons (top) and the baryons (bottom) for $\left(\kappa_{\mathrm{ud}}, \kappa_{\mathrm{s}}\right)=(0.13727,0.13640)$, $(0.13770,0.13640)$ and $(0.13781,0.13640)$ from the left. 


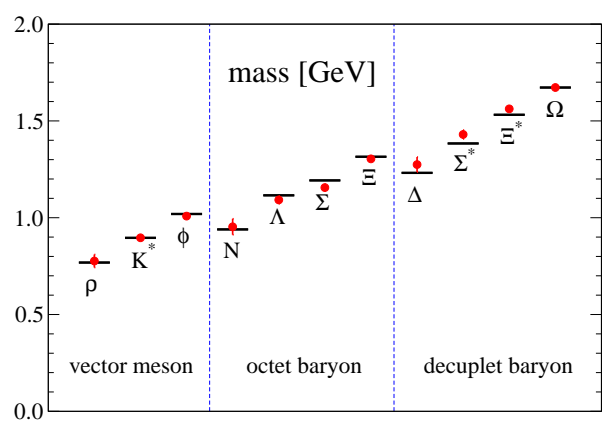

Figure 5: Light hadron spectrum extrapolated to the physical point (red circles) in comparison with the experimental values (black bars).

Figure 4 shows the hadron effective masses at $\kappa_{\mathrm{ud}}=0.13727,0.13770$ and 0.13781 . We observe clear plateau for the mesons except the $\rho$ meson at $\kappa_{\mathrm{ud}}=0.13781$ and also good signal for the baryons thanks to a large volume. Especially, the $\Omega$ baryon has a stable signal and a weak up-down quark mass dependence for our simulation parameters. Taking advantage of this virtue we choose the $\Omega$ baryon as input to determine the lattice cutoff. Combined with the additional inputs of $m_{\pi}$ and $m_{K}$ to determine the physical up-down and strange quark masses, we obtain $a^{-1}=2.176(31)$ $\mathrm{GeV}$. In this procedure we employ the SU(2) ChPT analyses for the quark mass dependences of $m_{\pi}$, $m_{K}, f_{\pi}$ and $f_{K}$ taking account of the finite size corrections evaluated at the one-loop level[8, 16]. For $m_{\Omega}$ we assume the linear quark mass depenedences. With the use of this cutoff we find that the lightest pseudoscalar meson mass we have reached is about $160 \mathrm{MeV}$. To obtain the vector meson masses and the baryon masses at the physical point we avoid the chiral analyses based on the heavy meson effective theory or the heavy baryon ChPT because of their poor convergences in the chiral expansions. We instead use linear chiral extraporations to the physical point. In Fig. 5 we compare the light hadron spectrum at the physical point with the experimental values. The largest discrepancy is at most $3 \%$, albeit errors are still not small for the $\rho$ meson, the nucleon and the $\Delta$ baryon. It should be also noted that our results contain possible $O\left(\left(a \Lambda_{\mathrm{QCD}}\right)^{2}\right)$ cutoff errors.

We calculate the bare quark masses using the axial vector Ward-Takahashi identity (AWI) defined by $a m^{\mathrm{AWI}}=\lim _{t \rightarrow \infty}\left\langle\nabla_{4} A_{4}^{\mathrm{imp}}(t) P(0)\right\rangle /(2\langle P(t) P(0)\rangle)$ where $A_{4}^{\mathrm{imp}}$ is the nonperturbatively $O(a)$-improved axial vector current [23]. Employing the perturbative renormalization factors $Z_{A}$ and $Z_{P}$ evaluated up to one-loop level [24, 25], we obtain

$$
m_{\mathrm{ud}}^{\overline{\mathrm{MS}}}(\mu=2 \mathrm{GeV})=2.527(47) \mathrm{MeV}, \quad m_{\mathrm{s}}^{\overline{\mathrm{MS}}}(\mu=2 \mathrm{GeV})=72.72(78) \mathrm{MeV} .
$$

The physical up-down quark mass is $30 \%$ smaller than our lightest one $m_{\mathrm{ud}}^{\overline{\mathrm{MS}}}(\mu=1 / a)=3.5 \mathrm{MeV}$ at $\left(\kappa_{\mathrm{ud}}, \kappa_{\mathrm{s}}\right)=(0.13781,0.13640)$. The results for the pseudoscalar meson decay constants are given by

$$
f_{\pi}=134.0(4.2) \mathrm{MeV}, \quad f_{K}=159.4(3.1) \mathrm{MeV}, \quad f_{K} / f_{\pi}=1.189(20)
$$

at the physical point with the perturbative $Z_{A}$. They are consistent with the experimental values within the errors. Our concern about the values for the quark masses and the pseudoscalar meson decay constants is the use of the perturbative renormalization factors which might cause sizable 
systematic errors. We are now calculating the nonperturbative $Z_{A}$ and $Z_{P}$ with the Schrödinger functional scheme.

\section{Acknowledgments}

Numerical calculations for the present work have been carried out on the PACS-CS computer at Center for Computational Sciences, University of Tsukuba. A part of the code development has been carried out on Hitachi SR11000 at Information Media Center of Hiroshima University. This work is supported in part by Grants-in-Aid for Scientific Research from the Ministry of Education, Culture, Sports, Science and Technology (Nos. 16740147, 17340066, 18104005, 18540250, 18740130, 19740134, 20340047, 20540248, 20740123, 20740139).

\section{References}

[1] PACS-CS Collaboration, S. Aoki et al., PoS (LAT2005) 111.

[2] PACS-CS Collaboration, A. Ukawa et al., PoS (LAT2006) 039.

[3] PACS-CS Collaboration, Y. Kuramashi et al., PoS (LAT2006) 029.

[4] Y. Kuramashi, PoS (LAT2007) 017.

[5] PACS-CS Collaboration, N. Ukita et al., PoS (LAT2007) 138.

[6] PACS-CS Collaboration, D. Kadoh et al. PoS (LAT2007) 109.

[7] Y. Kuramashi, these proceedings.

[8] S. Aoki et al., arXiv:0807.1661 [hep-lat].

[9] M. Lüscher, JHEP 0305 (2003) 052; Comput. Phys. Commun. 156 (2004) 209; ibid. 165 (2005) 199.

[10] A. Kennedy, Nucl. Phys. B (Proc. Suppl.) 140 (2005) 190.

[11] M. Hasenbusch, Phys. Lett. B519 (2001) 177.

[12] M. Hasenbusch and K. Jansen, Nucl. Phys. B659 (2003) 299.

[13] R. Brower, T. Ivanenko, A. Levi and K. Orginos, Nucl. Phys. B484 (1997) 353.

[14] M. Parks et al., SIAM J. Sci. Comput. 28 (2006) 1651.

[15] PACS-CS Collaboration, K-I. Ishikawa et al., PoS (LAT2006) 027.

[16] PACS-CS Collaboration, D. Kadoh et al., these proceedings.

[17] PACS-CS Collaboration, Y. Namekawa et al., these proceedings.

[18] CP-PACS and JLQCD Collaborations, S. Aoki et al., Phys. Rev. D73 (2006) 034501.

[19] Y. Iwasaki, preprint, UTHEP-118 (Dec. 1983), unpublished.

[20] CP-PACS and JLQCD Collaborations, T. Ishikawa et al., PoS (LAT2006) 181.

[21] CP-PACS and JLQCD Collaborations, T. Ishikawa et al., hep-lat/0704.193.

[22] J. C. Sexton and D. H. Weingarten, Nucl. Phys. B380 (1992) 665.

[23] CP-PACS/JLQCD and ALPHA Collaborations, T. Kaneko et al., JHEP 0704 (2007) 092.

[24] S. Aoki et al., Phys. Rev. D58, (1998) 074505.

[25] Y. Taniguchi and A. Ukawa, Phys. Rev. D58 114503 (1998). 